\title{
Long range channel characteristics through foliage
}

\author{
Nurul Afifah Binti Masadan, Mohamed Hadi Habaebi, Siti Hajar Yusoff \\ Department of Electrical \& Computer Engineering, International Islamic University Malaysia, 53100 KL, Malaysia
}

\begin{tabular}{l} 
Article Info \\
\hline Article history: \\
Received Mar 11, 2019 \\
Revised May 14, 2019 \\
Accepted May 29, 2019 \\
\hline
\end{tabular}

\section{Keywords:}

Foliage attenuation

Lorawan

Low-power wide-area

network (LPWAN)

Propagation model

\begin{abstract}
Long Range Low Power Wide Area Network (LoRa LPWAN) technology is unique and remarkable technology because of its long-range coverage, low power consumption and low cost system architecture. These features have allowed Lora LPWAN to become a favorable option for performing communication in most of IoT wireless applications. In this paper, the foliage effect has been studied in terms of attenuation and its overall contribution to the path-loss and link budget calculations. Specifically, 5 tree types were studied and their contribution to the path loss were quantified for different path crossings (e.g., trunk, tree-top and branches). The trees are Licuala Grandis, Mimusops Elengi, Mangifera Indica, Cyrtostachys Renda and Livistona Chinensis. Mimusops Elengi tree gave the strongest mean foliage attenuation accumulating up to $20 \mathrm{~dB}$, due to its big size and crown density. Trunks contribute even higher attenuation in comparison to tree-tops and branches. The Okumura/Hata, Log-normal shadowing and foliage models are used as references for this propagation models development in this paper. Our study showed that Okumura fails to capture the effect of foliage in an environment rich in trees and biodiversity. This demonstrates the need for considering the tropical environment where the characterization of foliage attenuation plays an important role in determining the propagation model path-loss and link budget needed for network design and planning.
\end{abstract}

Copyright $@ 2019$ Institute of Advanced Engineering and Science. All rights reserved.

\section{Corresponding Author:}

Nurul Afifah Binti Masadan,

Department of Electrical and Computer Engineering,

Kulliyyah of Engineering, International Islamic University Malaysia,

Jalan Gombak, Selangor, Malaysia.

Email: afifahdayne@gmail.com

\section{INTRODUCTION}

Over the last few years, there is an introduction to the Low Power Wide Area Network (LPWAN) technologies, causing industrial organization as well as academic association become interested with their capability performances $[1,2]$. LPWAN technologies including Sigfox, LoRa, Weightless and NB-IoT allows low cost with low power consumption wireless communications and are largely developed for M2M networking environment. Because of their characteristics, LPWAN technologies become considerably needed in the world of IoT.

LoRaWAN, which the word LoRa stands for long range wireless communication system, is one of the LPWAN technologies developed by Samtech Corporation that is designed for M2M and IoT networks [3,4]. It enables long range or wide coverage with an efficient power consumption as well as low cost communication system. With dynamic architecture and remarkable features, LoRaWAN is envisioned to facilitate or assist the multi-tenant networks in the development of smart city. LoRa can be referred to two distinct parts, i.e. the physical layer, patented by Semtech and the medium access control (MAC) layer protocol and network system architecture (LoRaWAN), designed and standardized by LoRa Alliance $[4,5]$.

The potency of LoRa networks in terms of power consumption, range and cost fulfill the demands of IoT making this technology implemented globally and become part of the most outstanding technologies. 
Accordingly, Malaysia also intend to commercialize city-wide LoRa network deployment. For now, the LoRa gateways have been installed in Klang Valley that covers only Kuala Lumpur, Petaling Jaya, Shah Alam and Port Klang.

As Malaysia has tropical environment, the propagation attenuation is different from other countries. Therefore, it is necessary to consider the tropical environment where the characterization of foliage attenuation is important in determining the path loss propagation model for network design.

\subsection{Existing propagation model}

The received signal strength (RSS) can decrease due to the effect of the reflection, precipitation, diffraction, penetration and scattering that are promoted by buildings or foliage. Therefore, the traits of common radio propagation models should be investigated and analyzed to indicate the way in which LoRa propagates and benchmark the performance of LoRa in different types of environments [6, 7].

\subsubsection{Okumura/Hata model}

Hata model is typically known as Okumura-Hata model as it is developed from the Okumura model, one of the radio propagation model that most of the people used to predict path loss in urban region because of its performance in accuracy and simplicity. However, the effects of urbanization degree or level are also not taken into account in this model.

By using Hata model, the effects of diffraction, reflection and scattering which are caused by the buildings or city formation as well as foliage can be comprehended. It identifies the overall signal attenuation by quantifying the 'obstacles effects' between the base station and end device and has become a standard for modern mobile radio systems. The path loss is different for each area, which for urban area the path loss can be calculated as

$$
L_{b}=69.55+26.16 \log \left(f_{c \mid M H z}\right)-13.82 \log h_{b}-a\left(h_{m}\right)+\left(44.9-6.55 \log h_{b}\right) \log \left(d_{\mid k m}\right)
$$

where $h_{b}$ and $h_{m}$ are base station antenna and mobile station antenna height (in meter) respectively, $\mathrm{f}_{\mathrm{c}}$ is the carrier frequency, $\mathrm{d}$ is the separation distance and $a\left(h_{m}\right)$ is the correction factor for mobile antenna height,

$$
a\left(h_{m}\right)=\left(1.1 \log \left(f_{c \mid M H z}\right)-0.7\right) h_{m}-\left(1.56 \log \left(f_{c \mid M H z}\right)-0.8\right)
$$

\subsubsection{Log-normal Shadowing}

In the coverage area, there might be some obstruction on the propagation path between base station and mobile station. The obstruction created by large hindrances or interferences like high-rise buildings and foliage environment result in instabilities of the receive signal power, i.e. shadowing the receive signal. This shadowing commonly modeled by log-normal random variable [8].

The log-normal shadowing path loss exponent (PLE) can be determined by using floating intercept technique, same method as the technique used to model large-scale shadow fading. The path loss (in $\mathrm{dB}$ ) at distance $d>d_{o}$ in the far field region can be expressed as

$$
P L_{d_{o} \rightarrow d}(d B)=P L\left(d_{o}\right)+10 n \log \left(\frac{d}{d_{o}}\right)+x \quad d_{f} \leq d_{o} \leq d
$$

where $\operatorname{PL}\left(d_{o}\right)$ is the path loss at free space reference distance $d_{o}$ (in meter) from the transmitter. $d(m)$ in this equation is the distance between transmitter and receiver while $\mathrm{n}$ is the path loss exponent (PLE). $X$ is a Gaussian distributed random variable (in $\mathrm{dB}$ ) with zero mean that is implemented only if the shadowing effect occur, with standard deviation $\sigma$.

\subsubsection{Vegetation and foliage model}

The presence of vegetation and foliage can cause shadowing effect in outdoor propagation. Loss can occur through scattering, diffraction, reflection, multipath dispersion when the radio wave travel in vegetation and foliage environment. The process in the wave propagation can be complex because of the structure of foliage which is formed by different type of leaves, trunks and branches [9-11]. The foliage attenuation differs based on the appearance of wind, humidity and the vegetation thickness.

It can be predicted that in the tropical vegetation region, the foliage attenuation will be higher compared to the attenuation in the vegetation region that is far away from the equator. It is because of the broader leaves of the trees in the tropical environment while the leaves in other region which is much cooler has needle-like leaves [12-13]. 


\section{RESEARCH METHOD}

In this measurement exercise which is conducted in Islamic International University of Malaysia (IIUM), Gombak, the main method in this project is a received signal strength indicator (RSSI) measurement. The equipment used to measure the (RSSI) are LoRa module (RF1276) and wire whip antenna (transceiver). The setup is very simple which the modules can just be plugged into a PC using USB adapter (PL2303) for the parameter configuration as the adapter can be used as a bridge for USB and UART port converter. To simulate the two LoRa module communication via two computers, SSCOM tool provided by the manufacturer is used. The equipment setup of RSSI measurement test is shown in Figure 1.
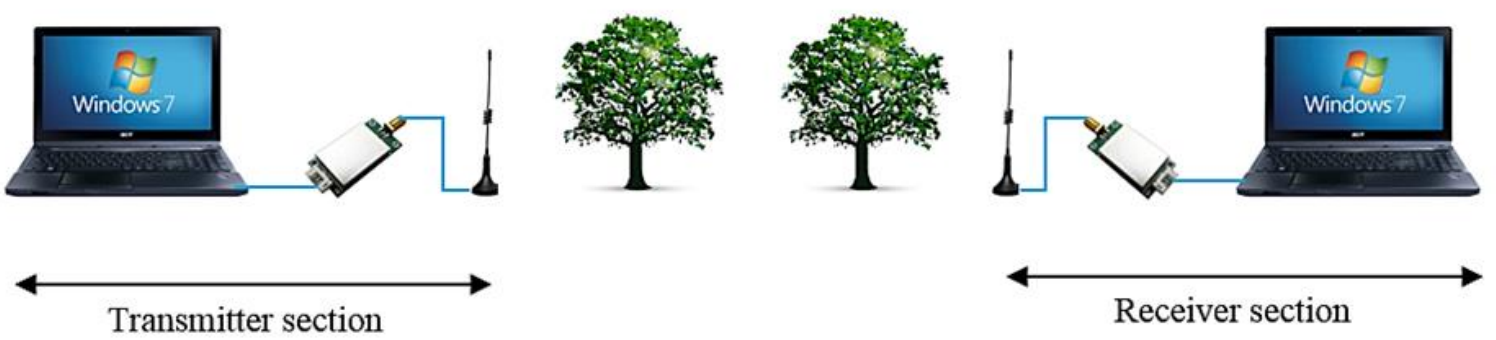

Figure 1. Equipment setup of the experiment [14]

The parameters used for LoRa measurement test is:

a. Frequency: $915 \mathrm{MHz}$

b. Factor: $1024(10)$

c. Bandwidth: $125 \mathrm{kHz}$

d. Baud rate: $9600 \mathrm{bps}$

e. Output power: $20 \mathrm{dBm}$

There are a few scenarios planned for the RSSI measurement exercise. All the scenarios take place in the university that has buildings as well as vegetation environment. LOS test is first done for horizontal and slant path. After that, the RSSI reading is taken across tree for both horizontal and slant (trunk, branches and treetop). Other than that, the coverage for LoRa communication is also determined by moving the receiver antenna around IIUM campus and then taking the reading from different locations in the campus.

\subsection{Line of sight (LOS) link or free space measurement}

For this scenario, the test is conducted at Saidina Hamzah Stadium located in the university. It has a very wide free space that is suitable for the measurement for both horizontal path and slant path. For the horizontal path, both the transmitter and receiver antennas are placed aligned with each other at the same height which is $0.65 \mathrm{~m}$ above the ground. On the other hand, for slant path test, the transmitter antenna is placed at height of about $4.5 \mathrm{~m}$ from ground level. Then the RSSI values are recorded when the receiver is moved incrementally until it reach about $25 \mathrm{~m}$ from the transmitter.

\subsection{Non-line of sight (NLOS) measurement}

Using the same equipment setup as LOS link test, the signal strength passes through the foliage is measured. The measurement for foliage propagation signal can be applied in two cases, one of them is measurement test for non-line of sight at slant path for single tree and the other measurement is at horizontal path across a single tree and a line of trees. Table 1 shows the tree measurement geometry for slant and horizontal path.

Besides that, propagation attenuation of the signal that passes through building also need to be investigated in this project. And for this reason, measurement test is conducted by moving the receiver antenna around IIUM campus to analyze the coverage of the LoRa propagation across the buildings and the vegetation as well. 


\begin{tabular}{|c|c|c|c|c|c|c|}
\hline Site & Geometry & Tree Species (scientific name) & Leaf shape & $\begin{array}{c}\text { Tree } \\
\text { height }\end{array}$ & $\begin{array}{c}\text { TX } \\
\text { antenna } \\
\text { height }\end{array}$ & $\begin{array}{c}\text { RX } \\
\text { antenna } \\
\text { height }\end{array}$ \\
\hline Tree 1 & Single tree (slant path) & Fan Palm (Licuala Grandis) & Fan/Palmate & $3.5 \mathrm{~m}$ & $6.8 \mathrm{~m}$ & $0.19 \mathrm{~m}$ \\
\hline Tree 2 & Single tree (slant path & Spanish Cherry (Mimusops Elengi) & Oval & $6.1 \mathrm{~m}$ & 10.3 & $0.19 \mathrm{~m}$ \\
\hline Tree 3 & Single tree (slant path & Mango Tree (Mangifera Indica) & Elliptical & $7.1 \mathrm{~m}$ & $11.0 \mathrm{~m}$ & $0.19 \mathrm{~m}$ \\
\hline Tree 4 & Single tree (slant path & Red Palm (Cyrtostachys Renda) & Pinnate & $1.65 \mathrm{~m}$ & $1.85 \mathrm{~m}$ & $0.19 \mathrm{~m}$ \\
\hline Tree 5 & $\begin{array}{l}\text { Single \& A line of } \\
\text { trees (horizontal path) }\end{array}$ & $\begin{array}{l}\text { Chinese Fan Palm } \\
\text { (Livistona chinensis) }\end{array}$ & Fan/Palmate & $\begin{array}{l}\text { About } \\
10 \mathrm{~m}\end{array}$ & $1.4 \mathrm{~m}$ & $1.4 \mathrm{~m}$ \\
\hline
\end{tabular}

\subsubsection{Single tree measurement at slant path}

4 different types of trees are used for this test to figure out the effects of the tree characteristics and parameters on the propagation signal. The RSSI is measured by passing the signal through single tree to emulate propagation through branches, trunk and treetop. For all the trees included in this scenario, RX antenna is put on the ground where the height of the antenna is just about $0.19 \mathrm{~m}$ whereas the TX antenna is put in different height for each of the tree depending on that particular tree height.

\subsubsection{Measurement at horizontal path}

For horizontal path, the measurement is conducted by surpassing the propagation through a single tree as well as a line of trees horizontally. For this measurement, the RSSI is measured across trunk as both antennas are placed at $1.4 \mathrm{~m}$ height above ground.

\subsubsection{Measurement around IIUM}

To determine how far the receiver can receive the signal from the transmitter, the measurement is carried out by moving the receiver around IIUM as the RSSI value could be affected by some attenuation that is produced by the buildings and vegetation environment in the university. The transmitter antenna is located at Level 3 Block C, Mahallah Halimatus Saadiah with height of $9.5 \mathrm{~m}$ and then the receiver antenna is moved around the campus for outdoor and indoor locations. The locations of both antennas are shown on map in Figure 2. Pink circle indicates the outdoor locations while orange square indicates indoor location.

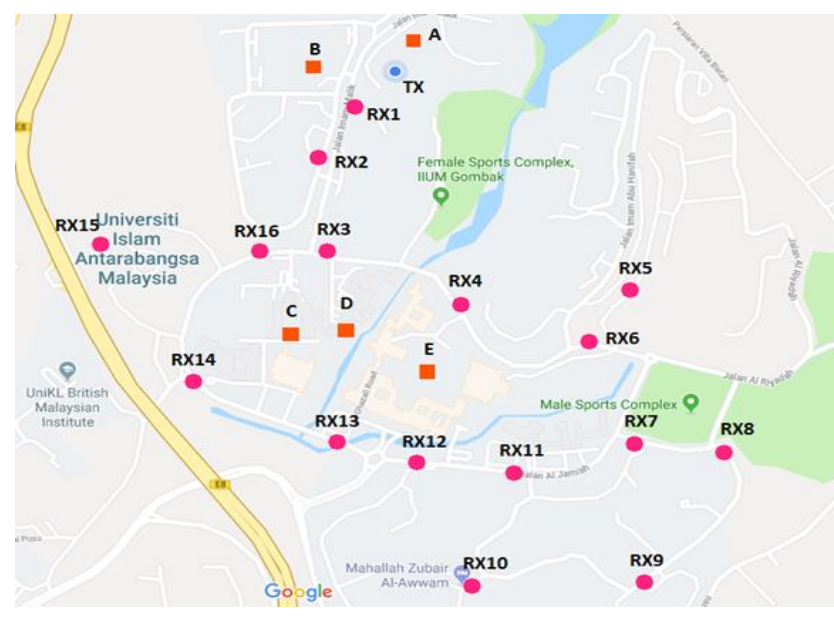

Figure 2. TX and RX location in IIUM Campus [14]

\subsection{Foliage Attenuation Calculation}

For LOS link measurement when there is no obstruction in between the antennas the free space path loss, $\mathrm{L}_{\mathrm{fs}}$ is calculated using formula in (1)

$$
L_{f s}=32.45+20 \log \left(f_{c \mid M H z}\right)+20 \log \left(d_{\mid k m}\right)
$$

where $\mathrm{f}_{\mathrm{c}}$ is frequency in $\mathrm{MHz}$ while $\mathrm{d}$ is the distance between transmitter and receiver in $\mathrm{km}$. Thus, the average received power, $\mathrm{P}_{\mathrm{fs}}(\mathrm{RX})$ can be calculated in (2) and (3) 


$$
\begin{aligned}
& P_{f S}(R X)=P_{t}+G_{t}+G_{r}-L_{f s} \\
& P_{f s}(R X)=P_{t}+G_{t}+G_{r}-32.45-20 \log \left(f_{c \mid M H z}\right)-20 \log \left(d_{\mid k m}\right)
\end{aligned}
$$

where $P_{t}$ is the transmitted power in $d B m$ and $G_{t}$ and $G_{r}$ are TX antenna gain and RX antenna gain respectively. For NLOS link measurement when there are trees and buildings as obstructions between the transmitter and receiver, the power received $\mathrm{P}_{\mathrm{obs}}(\mathrm{RX})$ is calculated as

$$
P_{o b s}(R X)=P_{t}+G_{t}+G_{r}-L_{f s}-L_{o b s}
$$

Therefore, the excess attenuation (A) formula is can be calculated as,

$$
A=P_{o b s}(R X)-P_{f s}(R X)
$$

\section{RESULTS AND ANALYSIS}

Based on the equation 4 , the path loss can be calculated by using the formula of

$$
L_{p}=P_{t}+G_{t}+G_{r}-P(R X)
$$

In this project, the tests used $20 \mathrm{dBm}$ as the transmitted power, and $5 \mathrm{dBi}$ gain for both transmitter and receiver antennas [15]. $\mathrm{P}(\mathrm{RX})$ is the received power (RSS value) measured for each test.

\subsection{LOS Link or Free Space Measurement}

For the measurement at horizontal path, it can be seen from Table 2 that the value of RSSI is varying from $-53 \mathrm{dBm}$ to $-74 \mathrm{dBm}$ when the distance between transmitter and receiver is increased 1 metre incrementally. At distance $15 \mathrm{~m}$ from the transmitter, the power received by the receiver is $-74 \mathrm{dBm}$ which is the lowest value recorded in this horizontal path test.

Table 2. LOS measurement results at horizontal path

\begin{tabular}{ccc}
\hline Distance between TX and RX & RSSI $(\mathrm{dBm})$ & Path loss $(\mathrm{dB})$ \\
\hline $1 \mathrm{~m}$ & -53 & 83 \\
$2 \mathrm{~m}$ & -59 & 89 \\
$3 \mathrm{~m}$ & -57 & 87 \\
$4 \mathrm{~m}$ & -60 & 90 \\
$5 \mathrm{~m}$ & -64 & 94 \\
$6 \mathrm{~m}$ & -62 & 92 \\
$7 \mathrm{~m}$ & -64 & 94 \\
$8 \mathrm{~m}$ & -63 & 93 \\
$9 \mathrm{~m}$ & -66 & 96 \\
$10 \mathrm{~m}$ & -67 & 97 \\
$11 \mathrm{~m}$ & -67 & 97 \\
$12 \mathrm{~m}$ & -69 & 99 \\
$13 \mathrm{~m}$ & -70 & 100 \\
$14 \mathrm{~m}$ & -69 & 99 \\
$15 \mathrm{~m}$ & -74 & 104 \\
\hline
\end{tabular}

On the other hand, for slant path measurement, the RSSI value fluctuates at distance $7 \mathrm{~m}$ until $25 \mathrm{~m}$ from the transmitter as shown in Table 3. The highest value is $-62 \mathrm{dBm}$ whereas the lowest value is $-80 \mathrm{dBm}$. From the results presented in the two tables, it is shown that the path loss are quite high for both horizontal and slant path. Path losses for horizontal path measurement vary from $83 \mathrm{~dB}$ for $1 \mathrm{~m}$ distance and $104 \mathrm{~dB}$ for $15 \mathrm{~m}$ distance. These results are high compared to when the free space path loss is calculated using formula in (4). For $1 \mathrm{~m}$ distance the free space path loss is estimated to be $32 \mathrm{~dB}$ while for $15 \mathrm{~m}$ distance the path loss is estimated to be $55 \mathrm{~dB}$. These results are showing that the difference between the theoretical path loss and measured path loss is quite big. 
Table 3. LOS measurement results at slant path

\begin{tabular}{cccc}
\hline Distance between TX and RX & $\emptyset$ (degree) & RSSI $(\mathrm{dBm})$ & Path loss $(\mathrm{dB})$ \\
\hline $6 \mathrm{~m}$ & 30 & -62 & 92 \\
$7 \mathrm{~m}$ & 29 & -69 & 99 \\
$8 \mathrm{~m}$ & 26 & -79 & 109 \\
$9 \mathrm{~m}$ & 23 & -71 & 102 \\
$10 \mathrm{~m}$ & 21 & -72 & 104 \\
$11 \mathrm{~m}$ & 19 & -74 & 110 \\
$12 \mathrm{~m}$ & 18 & -80 & 105 \\
$13 \mathrm{~m}$ & 16 & -75 & 102 \\
$14 \mathrm{~m}$ & 15 & -72 & 108 \\
$15 \mathrm{~m}$ & 14 & -78 & 108 \\
$16 \mathrm{~m}$ & 14 & -78 & 105 \\
$17 \mathrm{~m}$ & 13 & -75 & 103 \\
$18 \mathrm{~m}$ & 12 & -72 & 102 \\
$19 \mathrm{~m}$ & 11 & -73 & 104 \\
$20 \mathrm{~m}$ & 11 & -72 & 107 \\
$21 \mathrm{~m}$ & 10 & -74 & 105 \\
$22 \mathrm{~m}$ & 10 & -77 & 109 \\
$23 \mathrm{~m}$ & 10 & -75 & 110 \\
$24 \mathrm{~m}$ & 9 & -79 & -80 \\
$25 \mathrm{~m}$ & 9 & -10 & \\
\hline
\end{tabular}

\subsection{NLOS Link Measurement At Slant Path}

Table 4 shows the data statistics of single tree measurement at slant path where the propagation is emulated through trunk, treetop and branches. It can be observed that Tree 2 which is Mimusops Elengi gave the strongest mean foliage attenuation which is accumulating up to $20 \mathrm{~dB}$. The large attenuation produced by Tree 2 is because of its wide crown density and denser type of leaf. Besides that, among the three path crossings, trunk induced the highest attenuation for most of the tree. It is because of the scattering from lower region of the canopy and the diffraction loss caused by major fresnel zone blockage

Table 4. The attenuation of single tree measurement as slant path

\begin{tabular}{|c|c|c|c|c|c|c|}
\hline Tree & Path & $\varnothing$ (degree) & $\begin{array}{c}\text { LOS link RSSI } \\
(\mathrm{dBm})\end{array}$ & $\begin{array}{l}\text { NLOS link RSSI } \\
(\mathrm{dBm})\end{array}$ & $A(d B)$ & $\begin{array}{c}\text { Mean A } \\
(\mathrm{dB})\end{array}$ \\
\hline & a) Trunk & $28^{\circ}$ & -69 & -81 & 12 & \\
\hline \multirow[t]{3}{*}{ Tree 1} & b) Branches & $25^{\circ}$ & -79 & -80 & 1 & 7.7 \\
\hline & c) Treetop & $21^{\circ}$ & -72 & -82 & 10 & \\
\hline & a) Trunk & $33^{\circ}$ & -62 & -85 & 23 & \\
\hline \multirow[t]{3}{*}{ Tree 2} & b) Branches & $28^{\circ}$ & -69 & -94 & 25 & 19.7 \\
\hline & c) Treetop & $24^{\circ}$ & -71 & -82 & 11 & \\
\hline & a) Trunk & $31^{\circ}$ & -62 & -73 & 11 & \\
\hline \multirow[t]{3}{*}{ Tree 3} & b) Branches & $21^{\circ}$ & -72 & -78 & 6 & 8 \\
\hline & c) Treetop & $12^{\circ}$ & -72 & -79 & 7 & \\
\hline & a) Trunk & $33^{\circ}$ & -62 & -60 & 2 & \\
\hline \multirow[t]{2}{*}{ Tree 4} & b) Branches & $13^{\circ}$ & -75 & -63 & 12 & 9.3 \\
\hline & c) Treetop & $9^{\circ}$ & -79 & -65 & 14 & \\
\hline
\end{tabular}

\subsection{NLOS Link Measurement At Horizontal Path}

Based on the data in Table 5, as the separation distance is increased between the transmitter and receiver antennas, there is fluctuation of the RSSI value. This is because of the propagation path is blocked completely by the tree trunk which created considerable diffraction loss. It is proved that from the results obtained, the RSSI values measured are not merely effected by the separation distance as the values fluctuate from $-75 \mathrm{dBm}$ until $-99 \mathrm{dBm}$ when the receiver is incrementally moved further away from the transmitter.

Table 4. Data statistics for NLOS link measurement of single tree at horizontal path

\begin{tabular}{ccccc}
\hline Receiver & $\begin{array}{c}\text { Distance between } \\
\text { TX and RX }\end{array}$ & RSSI (dBm) & Path loss $(\mathrm{dB})$ & A $(\mathrm{dB})$ \\
\hline RX1 & $4.0 \mathrm{~m}$ & -81 & 111 & 21 \\
RX2 & $5.0 \mathrm{~m}$ & -74 & 104 & 10 \\
RX3 & $6.0 \mathrm{~m}$ & -88 & 118 & 26 \\
RX4 & $7.0 \mathrm{~m}$ & -79 & 109 & 15 \\
RX5 & $8.0 \mathrm{~m}$ & -78 & 108 & 15 \\
RX6 & $9.0 \mathrm{~m}$ & -99 & 129 & 33 \\
\hline
\end{tabular}


The data statistics shown in Table 6 infer that as the number of obstructing tree increases, the RSSI value decreases. This is because of the denser the obstruction, the higher the degradation of the received signal. Because of the signal is propagate into a trunk which is the thickest part of a tree, the difference between the RSSI for LOS link and NLOS link measurement measured is quite large compared to the RSSI measurement across other parts of tree as presented in slant path measurement result.

Table 5. Data statistics for NLOS link measurement of a line of trees at horizontal path

\begin{tabular}{ccccccc}
\hline Receiver & $\begin{array}{c}\text { \# of } \\
\text { tree }\end{array}$ & $\begin{array}{c}\text { Distance from } \\
\text { TX }\end{array}$ & $\begin{array}{c}\text { LOS link } \\
\text { RSSI }(\mathrm{dBm})\end{array}$ & $\begin{array}{c}\text { NLOS link } \\
\text { RSSI (dBm) }\end{array}$ & Path loss $(\mathrm{dB})$ & A (dB) \\
\hline RX1 & 1 & $4.0 \mathrm{~m}$ & -60 & -82 & 112 & 22 \\
RX2 & 2 & $8.8 \mathrm{~m}$ & -66 & -85 & 115 & 19 \\
RX3 & 3 & $12.9 \mathrm{~m}$ & -70 & -86 & 116 & 16 \\
RX4 & 4 & $16.6 \mathrm{~m}$ & -74 & -90 & 120 & 16 \\
\hline
\end{tabular}

Figure 3 shows the plotted graph for the attenuation, A $(\mathrm{dB})$ produced by single tree at horizontal path where the RSSI is measured at short distance with $4 \mathrm{~m}$ of initial separation distance between transmitter and receiver. From the data results, it is observed that the attenuation across the trunk of the tree which is Chinese Fan Palm (Livistona Chinensis) tree, fluctuates over the separation distances. The highest attenuation is at distance $9 \mathrm{~m}$ from the transmitter whereas the lowest attenuation is at distance $5 \mathrm{~m}$ from the transmitter.

By using the same tree species, a line of four tree measurement is conducted which the attenuation is plotted in graph of Figure 4. Based on the results, the attenuation is highest at the first tree and lowest at the last two trees. The attenuation is high initially and then decrease gradually after the second tree. It is because of the random scattering which is causing the gradual transition in attenuation. Thus, it can be determined that at small foliage depth, the attenuation will be higher compared to larger foliage depth.

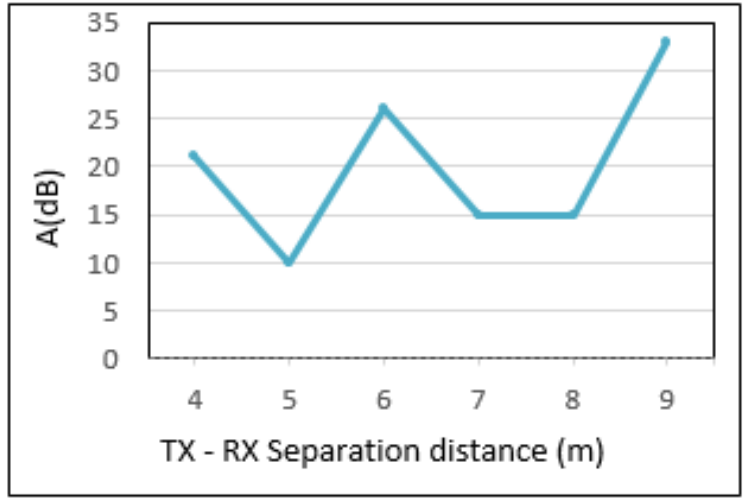

Figure 3. The plot of attenuation versus separation distance for single tree

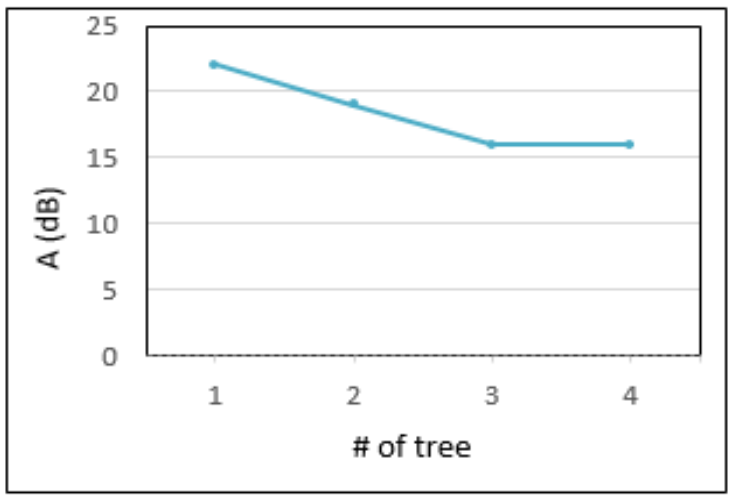

Figure 4. The plot of attenuation versus separation distance for a line of trees

\subsection{Path loss calculation using Okumura-Hata model}

The path loss obtained from the measurement test results are being compared with the path loss by Okumura-Hata model to quantify the attenuation that is caused by vegetation environment in the campus. By using the equation for urban area which in (1) and (2), the path loss is calculated to be compared with the path loss of the measurement around IIUM campus with the separation distance between transmitter and receiver that increases from $71 \mathrm{~m}$ to $1.4 \mathrm{~km}$. The comparison between the measured path loss and OkumuraHata path loss are tabulated in Table 7 and plotted in graph shown in Figure 5. 
Table 6. The comparison between measured path loss and Okumura-Hata path loss

\begin{tabular}{ccc}
\hline Distance between TX and RX $(\mathrm{m})$ & Experimental path loss $(\mathrm{dB})$ & Okumura-Hata Path loss $(\mathrm{dB})$ \\
\hline 71 & 145 & 89 \\
131 & 143 & 99 \\
181 & 156 & 104 \\
237 & 150 & 109 \\
472 & 153 & 121 \\
483 & 154 & 121 \\
522 & 157 & 122 \\
649 & 143 & 126 \\
765 & 146 & 129 \\
786 & 146 & 129 \\
803 & 151 & 129 \\
956 & 150 & 132 \\
977 & 146 & 133 \\
1050 & 153 & 134 \\
1053 & 138 & 134 \\
1180 & 150 & 136 \\
1310 & 158 & 138 \\
1400 & 150 & 139 \\
\hline
\end{tabular}

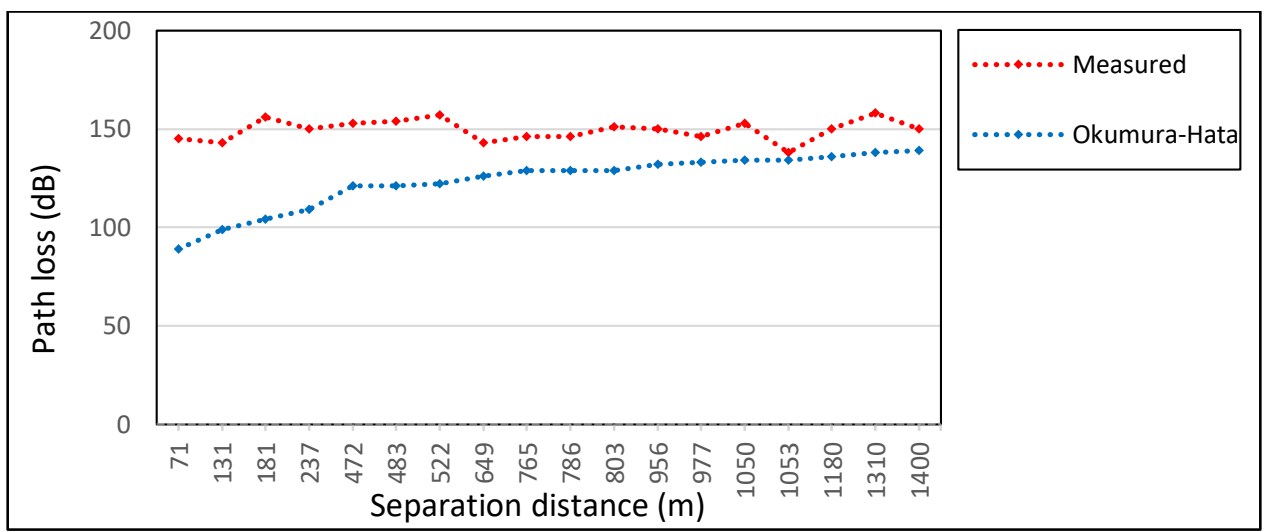

Figure 5. Comparison of measured path loss with Okumura-Hata path loss

Based on the plotted graph, the difference between the measured path loss and predicted path loss by Okumura-Hata model is quite large. The propagation attenuation in Malaysia might be bigger than other country because of the weather or the medium in which the signal propagation travel. Although there is significant difference in path loss, the Okumura-Hata model can be modified to predict the path loss in the campus. The attenuation produced by buildings and foliage could be added in order to predict the path loss accurately.

\subsection{Path loss exponent (n)}

Based on the plotted graph in Figure 6, the difference between the measured path loss and predicted path loss by Okumura-Hata model is quite large. The propagation attenuation in Malaysia might be bigger than other country because of the weather or the medium in which the signal propagation travel. Although there is significant difference in path loss, the Okumura-Hata model can be modified to predict the path loss in the campus. The attenuation produced by buildings and foliage could be added in order to predict the path loss accurately. 


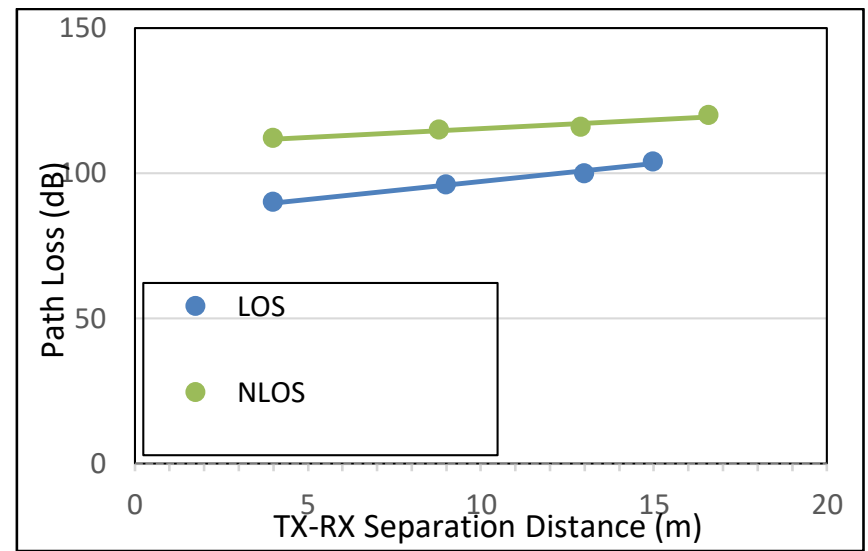

Figure 6. The plot of propagation path loss with separation distance

It is found that the $\mathrm{n}$ values for LOS quite high compared to the ideal value of $\mathrm{n}$ for free space which is 2. For NLOS, the value of $\mathrm{n}$ is 4.81 which indicates a $48.1 \mathrm{~dB}$ attenuation per decade while standard deviation of $17.51 \mathrm{~dB}$ corresponds to the shadowing effect produced by foliage. This idiates that foliage loss generated by line of trees are larger than free space propagation and should be taken into account.

\section{CONCLUSION}

This paper presents the measurement tests of three different scenarios for both LOS and NLOS links conducted in the campus. For LOS link test, the RSSI values obtained are quite low than expected. It is because of the big attenuation of the signal produced in the campus which has tropical climate environment. For NLOS link, the attenuation is larger as there are obstructions in between the transmitter and receiver. The obstruction leads to the diffraction, scattering and reflection of the signal which result in big attenuation. Five trees included in this study show the different characterization of foliage attenuation due to crown density and type of leaf. In addition to that, propagation through trunk gives highest foliage attenuation because of the diffraction loss caused by major fresnel zone. After some comparisons have been made, it can be determined that Okumura fails to capture the effect of foliage in IIUM campus that is in vegetation region. The model can be modified by adding the attenuation that is obtained experimentally to the path loss prediction formula of Okumura-Hata model.

\section{ACKNOWLEDGEMENTS}

This work is conducted at the IOT and Wireless Communication Lab and is supported by Malaysian Ministry of Higher Education (MOHE) Grant no. FRGS17-038-0604 and IIUM Publication RIGS grant no. P-RIGS19-003-0003.

\section{REFERENCES}

[1] J. Petäjäjärvi, K. Mikhaylov, R. Yasmin, M. Hämäläinen, and J. Iinatti, "Evaluation of LoRa LPWAN Technology for Indoor Remote Health and Wellbeing Monitoring," Int. J. Wirel. Inf. Networks, vol. 24, no. 2, pp. 153-165, 2017.

[2] Jetmir Haxhibeqiri, Floris Van den Abeele, Ingrid Moerman and Jeroen Hoebeke, "LoRa Scalability: A Simulation Model Based on Intererene Measurements," Sensors. 2017, 17(6), 1193; https://doi.org/10.3390/s17061193.

[3] M. Ghoraishi, J. Takada, and T. Imai, "Radio Wave Propagation Through Vegetation," Wave Propagation Theories and Applications. p. 20, 2013.

[4] N. A. B. Masadan, M. H. Habaebi and S. H. Yusoff, "LoRa LPWAN Propagation Channel Modelling in IIUM Campus," 2018 7th International Conference on Computer and Communication Engineering (ICCCE), Kuala Lumpur, 2018, pp. 14-19.

[5] L. Li, J. Ren and Q. Zhu, "On the application of LoRa LPWAN technology in Sailing Monitoring System," 2017 13th Annual Conference on Wireless On-demand Network Systems and Services (WONS), Jackson, WY, 2017, pp. 77-80.

[6] Famoriji J. Oluwole and Olasoji Y. Olajide, "Radio Frequency Propagation Mechanisms and Empirical Models for Hilly Areas", Canadian Journal on Electrical and Electronics Engineering, Vol. 4, No. 2, April 2013, PP.65-70. 
[7] S. Joshi and S. Sancheti, "Foliage loss measurements of tropical trees at $35 \mathrm{GHz}, " 2008$ International Conference on Recent Advances in Microwave Theory and Applications, Jaipur, 2008, pp. 531-532.

[8] M. H. Habaebi, I. J. Chowdhury, M. R. Islam, and N. A. B. Zainal, "Effects of shadowing on LoRa LPWAN radio links," Int. J. Electr. Comput. Eng., vol. 7, no. 6, pp. 2970-2976, 2017.

[9] N.A. Zainal, M.H. Habaebi, I. Chowdhury, M.R. Islam, "Cluttered Traffic Distribution in LoRa LPWAN,“ Indonesian Journal of Electrical Engineerig and Computer Science, 10(1), pp. 214-223, 2018.

[10] O. Georgiou and U. Raza, "Low Power Wide Area Network Analysis: Can LoRa Scale?," IEEE Wirel. Commun. Lett., vol. 6, no. 2, pp. 162-165, 2017.

[11] F. Adelantado, X. Vilajosana, P. Tuset-Peiro, B. Martinez, J. Melia-Segui and T. Watteyne, "Understanding the Limits of LoRaWAN," in IEEE Communications Magazine, vol. 55, no. 9, pp. 34-40, Sept. 2017.

[12] J. Petäjäjärvi, K. Mikhaylov, M. Pettissalo, J. Janhunen, and J. Iinatti, "Performance of a low-power wide-area network based on LoRa technology: Doppler robustness, scalability, and coverage," Int. J. Distrib. Sens. Networks, vol. 13, no. 3, p. 155014771769941, 2017.

[13] S. Hosseinzadeh, M. Almoathen, H. Larijani, and K. Curtis, "A Neural Network Propagation Model for LoRaWAN and Critical Analysis with Real-World Measurements," Big Data Cogn. Comput., vol. 1, no. 1, p. 7, 2017.

[14] N.A. Zainal, M.H. Habaebi, I. Chowdhury, M.R. Islam, "Cluttered Traffic Distribution in LoRa LPWAN," Indonesian Journal of Electrical Engineerig and Computer Science, 10(1), pp. 214-223, 2018.

[15] S. Choudhary and D. K. Dhaka, "Path loss Prediction Models for Wireless Communication Channels and its Comparative Analysis," International Journal of Engineering, Management \& Sciences (IJEMS). no. 3, pp. 38-43, 2015. 